\title{
Fatores-chave de sucesso no treinamento corporativo a distância via web*
}

\author{
Luiz Antonio Joia** \\ Mário de Fiqueiredo Cunha da Costa***
}

SumÁrio: 1. Introdução; 2. Referencial teórico; 3. Método de pesquisa; 4. Descrição dos casos; 5. Comparação de resultados; 6. Considerações finais.

SUMMARY: 1 . Introduction; 2 . Theoretical framework; 3 . Research method; 4. Case description; 5. Comparing results; 6. Final remarks.

Palavras-chave: treinamento corporativo na web; e-learning; instrução baseada na web; ensino a distância.

KEY WORDS: web-based corporate training; e-learning; web-based instruction; distance learning.

Treinamentos corporativos a distância via web vêm se tornando cada vez mais freqüentes, à medida que as empresas precisam continuamente capacitar seus profissionais a um custo acessível. No entanto, de um modo geral, as organizações não sabem quais são os fatores-chave de sucesso para iniciativas dessa natureza. Assim, este artigo investiga alguns fatores críticos de sucesso associados a esses empreendimentos digitais. Para tal, o método de estudo de casos múltiplos divergentes é utilizado, analisandose dois treinamentos corporativos na web realizados por uma grande empresa multinacional - um considerado caso de sucesso e outro de fracasso.

\footnotetext{
* Artigo recebido em out. 2006 e aceito em fev. 2007.

** Doutor em ciências em engenharia de produção e mestre em ciências em engenharia civil pela Coppe/UFRJ, mestre em management studies pela Oxford University e engenheiro de fortificação e construção pelo Instituto Militar de Engenharia (IME). Professor da Ebape/FGV e da Faculdade de Engenharia da Uerj. Endereço: Praia de Botafogo, 190, sala 526 - CEP 22250900, Rio de Janeiro, RJ, Brasil. E-mail: luiz.joia@fgv.br.

*** Mestre em administração pela Ebape/FGV. Projetista de sistemas sênior na IBM. Endereço: Praia de Botafogo, 190, sala 526 - CEP 22250-900, Rio de Janeiro, RJ, Brasil. E-mail: mariofcosta@uol.com.br.
} 
A partir da comparação dos resultados obtidos, pela análise quantitativa dos dados coletados usando-se regressões bi e multivariadas, assim como testes de comparação de médias, e vis-à-vis o frame teórico adotado para avaliação de treinamentos na web, pode-se concluir que "orientação dos objetivos", "motivação dos alunos" e "suporte metacognitivo" foram os três fatores críticos de sucesso encontrados.

\section{Key factors for the success of web-based corporate training}

Web-based corporate training has become more frequent as companies continuously need to train their employees under budgetary constraints. However, most companies do not know which are the key factors for these endeavors' success. This article investigates some critical success factors associated with these digital enterprises, using the multiple case study method is used, whereby two cases with opposite outcomes - a success and a failure - are analyzed. Comparing these two cases developed within a large multinational company by using quantitative data analysis based on bi and multivariate linear regressions, as well as comparison of averages, and vis-à-vis the theoretical framework adopted to assess web-based trainings, the study concludes that "goal orientation", "student motivation", and "metacognitive support" are three critical factors in corporate webbased training.

\section{Introdução}

Atualmente, onde a dinâmica de mudanças é muito grande, seja por orientação estratégica, por necessidade de adaptação a novas exigências do mercado, ou ainda, por necessidade de adequação à legislação vigente, é fundamental para as empresas obter agilidade, baixo custo e alto retorno sobre seus investimentos em capacitação. Por outro lado, o alto custo de programas de treinamento corporativos presenciais, associado a dificuldades logísticas para sua operacionalização e a rapidez crescente com que o seu conteúdo atinge a obsolescência, representam grandes desafios para sua viabilização.

É nesse cenário de enorme rapidez de mudanças, volume crescente de informações e demanda crescente por programas de capacitação que surge o treinamento corporativo a distância baseado na web, também chamado de e-learning. A tecnologia da internet consegue solucionar grande parte dos problemas e limitações das tecnologias até então utilizadas, tornando viável a implementação de treinamentos corporativos a distância (Rosemberg, 2001). 
Mesmo sendo um fator fundamental para a viabilização desses programas, a tecnologia por si só não fornece qualquer garantia de sucesso aos programas de treinamento, devendo ser associada a aspectos pedagógicos e didáticos relacionados aos mesmos. As características específicas de cada programa de treinamento devem ser cuidadosamente avaliadas e consideradas tão relevantes quanto os custos de implementação durante o processo de tomada de decisão (Clark, 1983).

A maior parte da literatura disponível sobre treinamentos corporativos a distância via web está voltada para os aspectos técnicos relacionados à sua implementação. As vantagens competitivas e a redução de custos associadas à utilização dessa tecnologia são enaltecidas, a ponto de alguns afirmarem que, em breve, não existirão mais salas de aula "tradicionais" (Abuhejleh, 2002). Apesar dessas avaliações, a construção de programas de treinamento a distância via web não é uma tarefa trivial, possuindo um conjunto ainda não bem consolidado de fatores-chave de sucesso (Carey, Peerenboom e Lytwyn, 1998; Penuel e Roschelle, 1999).

Este artigo investiga esses fatores, pela análise de dois distintos programas de treinamento a distância via web, com a seguinte pergunta de pesquisa: "Quais os fatores críticos de sucesso para a construção de programas corporativos de treinamento a distância baseados na web?".

A seguir, será feita uma especificação mais detalhada do referencial teórico adotado. Depois, será analisado o método de pesquisa utilizado neste artigo. A partir daí, serão apresentados dois estudos de caso de treinamento a distância na web desenvolvidos pela mesma empresa - um considerado um sucesso e outro, um fracasso - , analisando-se, para cada um deles, os fatores que conduziram aos resultados finais. Por fim, será feito um trabalho comparativo dos casos estudados, evidenciando-se os fatores que influenciaram esses processos, de modo a se poder discutir os resultados encontrados e apresentar algumas observações conclusivas.

\section{Referencial teórico}

Para analisar os aspectos teóricos relacionados ao treinamento a distância, é necessário abordar três assuntos inter-relacionados: psicologia, educação e tecnologia da informação (Wilhelmsen, Åsmul e Meistad, 1998). Mais especificamente, é necessário considerar as principais abordagens pedagógicas e os aspectos da utilização da tecnologia da informação como forma de aplicação das mesmas. 


\section{Abordagens pedagógicas}

No que tange a abordagens pedagógicas, serão analisados tangencialmente os dois paradigmas que se tornaram fundamentalmente influentes a partir do século XX. Eles não incluem somente a visão de como se dá o aprendizado, mas oferecem também uma visão sobre o que é o conhecimento - basicamente, se o conhecimento existe em uma forma absoluta, ou se é algo construído e relativo. Essas duas abordagens são comumente denominadas behaviorismo e construtivismo (Wilhelmsen, Åsmul e Meistad, 1998).

Como dito, a distinção básica entre behaviorismo e construtivismo está no conceito de conhecimento. Para os behavioristas, o conhecimento é passivo - respostas automáticas para fatores externos - , enquanto para os construtivistas, o conhecimento é visto como uma entidade construída por cada estudante, ao longo do processo de aprendizado. O conhecimento, sob a ótica construtivista, não tem uma característica absoluta como no behaviorismo, não podendo, dessa forma, ser simplesmente passado de uma pessoa para outra (Wilhelmsen, Åsmul e Meistad, 1998).

Para este artigo, os aspectos mais importantes da abordagem behaviorista são os conceitos de que o estudante deve se adaptar ao ambiente, e de que aprender é um processo passivo, no qual não existe um tratamento explícito ou interesse no processo mental. $\mathrm{O}$ estudante, nesse caso, meramente responde a demandas do ambiente (estímulos). O conhecimento é, então, visto como algo absoluto e imutável (ver, por exemplo, Skinner, 1968; Wilhelmsen, Åsmul e Meistad, 1998).

Assim, uma abordagem behaviorista para um determinado treinamento funciona bem, caso ele possua objetivos claramente definidos e seus resultados sejam facilmente mensuráveis. Como exemplo poderiam ser apresentados os vídeos de treinamento do Exército americano, utilizados na II Guerra Mundial, para tarefas repetitivas como montar um rifle (Rosemberg, 2001:20).

Ao contrário dos behavioristas, os teóricos do construtivismo acreditam que o aprendizado é um processo ativo. O construtivismo se baseia no conceito de que os estudantes constroem seu próprio conhecimento, em vez de o professor passar as informações e o conhecimento aos alunos (ver, por exemplo, Piaget, 1952; Papert, 1993).

Para os construtivistas, o plano de ensino deve ter sempre ênfase no estudante - em vez de no conteúdo e formato do programa - e no instrutor (University of Dayton, 2003). Passar-se-ia, dessa forma, de um modelo onde o instrutor é o centro do programa de ensino, para um modelo onde o estudante é o centro. 


\section{e-learning e treinamento corporativo na web}

Definir e-learning não é tarefa fácil. Assim como diversos outros conceitos relacionados à internet (e-commerce, e-business, e-collaboration etc.), este é um conceito novo, que unifica em um só termo diversos conceitos distintos.

A American Society for Training and Development (ASTD) define $e$ learning como um termo que cobre uma ampla variedade de aplicações e processos, como web-based training (WBT), computer-based training (CBT), salas de aula virtuais, e colaboração digital, objetivando permitir o treinamento a distância por meios eletrônicos. Inclui a disponibilização de conteúdo via internet, fitas de áudio e vídeo, difusão por satélite, TV interativa, CD-ROM e outras mídias.

Nota-se, portanto, que apesar de possuir uma nomenclatura que o associa com a internet, o e-learning não se restringe ao uso da web para disseminação de programas de treinamento.

Freqüentemente, os departamentos de uma empresa são orientados a desenvolver iniciativas de treinamento via web. Esses direcionamentos são, algumas vezes, orientados por um imperativo tecnicista, isto é, a necessidade de se utilizar a tecnologia internet per se. Em algumas organizações, o treinamento baseado na web foi criado meramente para justificar os custos da intranet corporativa (Powell, 2000). Conforme já comentado neste artigo, o uso da tecnologia per se não justifica a implementação de nenhuma forma de treinamento (Rosemberg, 2001; Bates, 1995; Kay, Dodd e Sime, 1970).

Para criar programas de treinamento corporativos e determinar seus objetivos, é preciso definir as necessidades dos indivíduos (empregados) e as necessidades do negócio (empresas) (Dick e Carey, 1996; Kay, Dodd e Sime, 1970; Mager, 1972; Sancho, 1998).

\section{Avaliação de programas de e-learning}

Para conduzir a análise comparativa de diferentes programas de educação a distância, é necessário adotar um modelo específico. Para este artigo, será utilizado o modelo proposto por Reeves e Reeves (1997). Martin (1998) e Joia (2001) propõem o uso desse modelo para a identificação e avaliação de 10 diferentes dimensões envolvidas nos treinamentos via web.

É importante salientar que o modelo de Reeves e Reeves (1997) não se propõe a avaliar o resultado de um programa de treinamento na web, nem tampouco prever seu sucesso ou fracasso. O objetivo desse modelo é caracterizar os diferentes aspectos desse tipo de programa (Reeves, 1996). 
Cada uma das 10 dimensões propostas por Reeves e Reeves (1997) é representada por um continuum, tendo em cada um de seus extremos as diferentes orientações possíveis. Cada uma dessas dimensões apresenta orientações específicas, mas todas são relacionadas com uma orientação básica que vai do behaviorismo ao construtivismo (Joia, 2001). (1997).

A seguir, são apresentadas as 10 dimensões propostas por Reeves e Reeves

\section{Filosofia pedagógica}

A primeira dimensão do modelo proposto trata da base pedagógica sobre a qual será montado o conteúdo do treinamento. Seguindo a definição proposta por diversos autores (Rieber, 1992; Duffy e Jonassen, 1992; Papert, 1993), Reeves (1996) define os extremos dessa dimensão como instrutivismo e construtivismo.

Os instrutivistas salientam a importância de metas e objetivos definidos para o programa de treinamento (sem levar em consideração o estudante). Joia (2001:9) esclarece que, nesse extremo, existe pouca ênfase nos estudantes, que são vistos como recipientes vazios a serem preenchidos pelo aprendizado. Nota-se, portanto, estreita relação entre essa abordagem e os preceitos behavioristas.

Os construtivistas, por outro lado, enfatizam a importância das intenções, experiências e estratégias cognitivas dos estudantes. De acordo com essa filosofia pedagógica, os estudantes constroem diferentes estruturas cognitivas baseadas em seus conhecimentos prévios e no que experimentam nos diferentes ambientes de aprendizado (Joia, 2001; Reeves, 1997). Assim como proposto por Piaget (1952), Reeves (1997) salienta que os estudantes, ao serem expostos a informações que podem entrar em conflito com suas construções mentais existentes, atingem o equilíbrio cognitivo pela reconstrução de conceitos, esquemas e modelos mentais, e outras estruturas cognitivas. Em vez de serem vistos como recipientes vazios, os estudantes são vistos como indivíduos repletos de conhecimento, atitudes e motivação (Joia, 2001; Reeves, 1997).

\section{Teoria de aprendizado}

Essa dimensão é caracterizada pela definição da teoria de aprendizado utilizada como base para a montagem do treinamento, tendo o behaviorismo num dos extremos do continuum e o cognitivismo no outro. 
Reeves (1997) observa que, apesar da atual corrente contrária ao behaviorismo, ele continua a ser a teoria básica utilizada para muitas formas de treinamento baseado em computador. Conforme já mencionado neste artigo, os behavioristas acreditam que os fatores importantes no aprendizado não são os estados internos da mente que podem ou não existir, mas o comportamento (behavior) que pode ser diretamente observado (Skinner, 1968). A instrução, portanto, consiste primariamente em moldar os comportamentos desejados com estímulos, respostas, feedbacks e reforços. Joia (2001) exemplifica: um estímulo é oferecido (uma pequena apresentação de conteúdo, por exemplo), uma resposta é obtida (normalmente com uma pergunta ou teste), o feedback é fornecido de acordo com a correção das respostas, e reforço positivo é fornecido para as respostas corretas.

Sem ignorar o comportamento (behavior), por outro lado, os cognitivistas dão muito maior ênfase aos estados internos da mente (Reeves, 1997). Uma taxonomia cognitiva para esses estados internos da mente inclui proposições simples, esquemas, regras, habilidades e modelos mentais (Joia, 2001). Por fim, os cognitivistas definem que diferentes estratégias devem ser utilizadas em todos os ambientes de aprendizado, dependendo do tipo de conhecimento a ser criado pelo estudante.

\section{Orientação dos objetivos}

A orientação dos objetivos de um programa de treinamento pode variar entre ser eminentemente específica (por exemplo, seguir um protocolo rígido para atendimento médico de emergência), até ser completamente genérica (por exemplo, aprender a apreciar arte moderna) (Reeves, 1996).

Cole (1992) enfatiza a relevância dos diferentes tipos de objetivos na construção de um programa de treinamento, ao salientar que alguns tipos de conhecimento são idealmente apresentados aos estudantes de forma direta e objetiva, enquanto outros demandam formas mais indutivas de aprendizado (realidade virtual, por exemplo).

\section{Orientação das tarefas}

O contexto do aprendizado é de extrema importância para os adultos (Merriam, 1993). Nesse sentido, a orientação das tarefas apresentadas aos estudantes pode variar do extremo absolutamente acadêmico até o absolutamente autêntico. 
Um treinamento acadêmico, por exemplo, possui um alto grau de dependência da sua capacidade de fazer com que os estudantes desenvolvam exercícios acadêmicos tradicionais, enquanto programas com uma característica autêntica levariam os estudantes a realizar atividades práticas, situando a prática e o feedback no contexto de situações reais (Joia, 2001).

Reeves (1997) salienta que se conhecimentos, habilidades e atitudes são assimilados no contexto prático, eles serão utilizados sempre que esse mesmo contexto, ou contextos similares, seja vivenciado pelos estudantes.

\section{Fonte de motivação}

A motivação é um fator primário em qualquer teoria ou modelo de aprendizado (Amabile, 1993; Carroll, 1968).

No modelo utilizado neste artigo, a motivação pode variar entre completamente extrínseca (vindo de fora do ambiente de aprendizado) até totalmente intrínseca (integralizada ao ambiente de aprendizado).

A instrução com motivação intrínseca é muito elusiva, independentemente de como é disponibilizada para o estudante. Virtualmente, todas as novas abordagens de ensino trazem a promessa de serem mais motivadoras do que as anteriores. A multimídia é o mais recente sistema de aprendizado interativo e, supostamente, motiva automaticamente os estudantes, simplesmente por integrar música, voz, vídeo, figuras, texto, animação, e uma interface amigável via computador (Reeves, 1997). Na prática, os aspectos motivacionais precisam ser, inevitavelmente, considerados no desenvolvimento de conteúdo multimídia, tanto quanto para qualquer outro tipo de conteúdo (Reeves, 1999).

\section{Papel do professor}

O continuum do papel do professor varia do didático ao facilitativo.

O papel didático pode ser associado aos tradicionais professores em salas de aula, que apresentam conteúdo aos estudantes. Nos programas onde a exposição dos professores é uma estratégia instrucional apropriada, os treinamentos por computador podem ser utilizados para auxiliar, reforçar e estender as suas apresentações (Reeves, 1997).

Por outro lado, quando o professor atua no papel de facilitador, a responsabilidade cognitiva passa para os estudantes, à medida que eles passam a ser responsáveis por julgar os padrões das informações, organizar dados, cons- 
truir perspectivas alternativas, e apresentar novos conhecimentos. Neste caso, os professores atuam como mentores e tutores deste processo (Reeves, 1997).

\section{Suporte metacognitivo}

O suporte metacognitivo se refere ao conhecimento, por parte do estudante, dos objetivos do treinamento, da sua habilidade para planejar e avaliar as estratégias de aprendizado, e da sua capacidade de monitorar seu progresso e ajustar seu comportamento para acomodar suas necessidades (Reeves, 1999:7). Neste sentido, esta dimensão varia do suporte metacognitivo não implementado ao integral.

A recapitulação das estratégias dos estudantes em qualquer ponto do processo de resolução de problemas, assim como a construção de portfólios baseados na web (ver Campbell et al., 2000), são exemplos de como o suporte metacognitivo pode ser implementado em programas de treinamento baseados na web (Joia, 2001).

\section{Aprendizado colaborativo}

De forma similar ao suporte metacognitivo, a dimensão de aprendizado colaborativo varia do totalmente não implementado ao integral. Essa dimensão refere-se às estratégias instrucionais nas quais os estudantes trabalham em pares, pequenos grupos ou, até mesmo, grandes grupos para atingirem objetivos comuns (Reeves, 1999:7; Joia, 2001).

Uma vez utilizado um design instrucional apropriado, dois ou mais estudantes trabalhando juntos via web podem ser mais produtivos do que um estudante isolado, uma vez que as interações entre eles podem ter mais influência no seu processo de aprendizado do que a interação entre eles e o conteúdo do programa (Reeves, 1999:7).

\section{Sensibilidade cultural}

Todos os sistemas de aprendizado têm implicações culturais (Joia, 2001). Para medir essa dimensão, Reeves (1997) propõe um continuum que varia da sensibilidade cultural absolutamente não implementada até a totalmente integralizada ao conteúdo e formato do programa de treinamento. 
Quando a sensibilidade cultural não é implementada, o treinamento é desenvolvido sem qualquer consideração pelas diferenças culturais e pela diversidade dos estudantes. Por outro lado, quando integralmente considerada, o conteúdo e formato do programa de treinamento devem considerar esses aspectos (Joia, 2001).

Joia (2001) salienta que é improvável que um treinamento via web possa ser desenhado de forma a se adaptar a todas as normas culturais existentes, mas recomenda que seja construído de forma a ser o mais sensível possível à cultura vigente.

\section{Flexibilidade estrutural}

A flexibilidade estrutural dos programas de treinamento baseados na web pode variar de estruturas fixas até completamente abertas (Joia, 2001).

Os sistemas fixos são usualmente limitados a locais específicos, com tempo determinado para cada atividade. A web fornece oportunidades para o desenvolvimento de estruturas abertas, mas, ainda assim, podem existir componentes fixos nos programas de treinamento (Joia, 2001).

\section{Consolidação das dimensões de avaliação de treinamento na web}

O quadro apresenta as 10 dimensões de análise dos programas de treinamento apresentadas neste artigo. Para cada uma das dimensões (coluna central da tabela) são definidos os dois extremos do continuum correspondente.

Conforme será detalhado no método de pesquisa, a avaliação da percepção de usuários e gestores dos programas de treinamento analisados, quanto a cada uma das 10 dimensões propostas neste modelo, levará à identificação dos seus fatores-chave de sucesso.

Dimensões para avaliação de programas de treinamento a distância

\begin{tabular}{lcr}
\hline$\leftarrow$ & Dimensão & $\rightarrow$ \\
\hline Instrutivismo & Filosofia pedagógica & Construtivismo \\
Conhecimento pertence ao instrutor & $0-10$ & Conhecimento como uma \\
Behaviorista & Teoria de aprendizado & construção na mente do estudante \\
Ênfase no comportamento observável & $0-10$ & Eognitivista \\
Específica & Orientação dos objetivos nos estados internos da mente \\
Instrução direta com foco em um & $0-10$ & Genérica \\
comportamento desejado & & Simulações com mais de uma \\
solução para um problema
\end{tabular}




\begin{tabular}{|c|c|c|}
\hline$\leftarrow$ & Dimensão & $\rightarrow$ \\
\hline $\begin{array}{l}\text { Acadêmica } \\
\text { Ênfase em exercícios acadêmicos } \\
\text { tradicionais }\end{array}$ & $\begin{array}{c}\text { Orientação das tarefas } \\
0-10\end{array}$ & $\begin{array}{r}\text { Autêntica } \\
\text { Ênfase em exercícios fora do alcance } \\
\text { do estudante, em conjuntos autênticos }\end{array}$ \\
\hline $\begin{array}{l}\text { Extrínseca } \\
\text { Motivação independente do } \\
\text { estudante e do ambiente de } \\
\text { aprendizado }\end{array}$ & $\begin{array}{l}\text { Fonte de motivação } \\
\qquad 0-10\end{array}$ & $\begin{array}{r}\text { Intrínseca } \\
\text { Motivação a partir do estudante e } \\
\text { do ambiente de aprendizado }\end{array}$ \\
\hline $\begin{array}{l}\text { Didática } \\
0 \text { instrutor é o repositório do } \\
\text { conhecimento }\end{array}$ & $\begin{array}{l}\text { Papel do professor } \\
\quad 0-10\end{array}$ & $\begin{array}{r}\text { Facilitativa } \\
0 \text { professor é um facilitador do } \\
\text { estudo, um guia para os estudantes }\end{array}$ \\
\hline $\begin{array}{l}\text { Não implementado } \\
\text { Sem monitoramento de progresso } \\
\text { dos estudantes nem ajustes às } \\
\text { necessidades individuais }\end{array}$ & $\begin{array}{l}\text { Suporte metacognitivo } \\
0-10\end{array}$ & $\begin{array}{l}\text { Integral } \\
\text { Com completo monitoramento de } \\
\text { progresso dos estudantes e ajustes } \\
\text { às necessidades individuais }\end{array}$ \\
\hline $\begin{array}{l}\text { Não implementado } \\
\text { Estudantes trabalham } \\
\text { individualmente }\end{array}$ & $\begin{array}{c}\text { Aprendizado colaborativo } \\
0-10\end{array}$ & $\begin{array}{r}\text { Integral } \\
\text { Estudantes trabalham em pares } \\
\text { ou pequenos grupos }\end{array}$ \\
\hline $\begin{array}{l}\text { Não implementada } \\
\text { Diferenças culturais não } \\
\text { são consideradas }\end{array}$ & $\begin{array}{l}\text { Sensibilidade cultural } \\
\qquad 0-10\end{array}$ & $\begin{array}{r}\text { Integral } \\
\text { Programa adaptado às diferenças } \\
\text { culturais dos estudantes }\end{array}$ \\
\hline $\begin{array}{l}\text { Fixa } \\
\text { Programa limitado a lugares e } \\
\text { horários específicos }\end{array}$ & $\begin{array}{l}\text { Flexibilidade estrutural } \\
\qquad 0-10\end{array}$ & $\begin{array}{r}\text { Aberta } \\
\text { Programa não limitado a lugares e } \\
\text { horários específicos }\end{array}$ \\
\hline
\end{tabular}

\section{Método de pesquisa}

Para este trabalho de pesquisa, foi adotado o método de estudo de casos múltiplos, como descrito e abordado por Yin (1994).

Estudos de casos são particularmente adequados para responder a perguntas do tipo: "Como?" e "Por quê?", sendo, também, especialmente úteis para geração e construção de teorias, onde poucos dados ou teorias existem (Yin, 1994), o que corresponde à situação em questão. Permitem, também, ao pesquisador, o uso do "oportunismo controlado", de modo a responder, de forma flexível, a novas descobertas feitas quando da coleta de novos dados (Eisenhardt, 1989).

Apesar de predominantemente exploratório, este artigo, ao tentar identificar uma relação causal entre as características dos programas de treinamento 
analisados e seus respectivos resultados, apresenta, também, características explanatórias. Estudos de casos explanatórios ou causais são úteis para avaliar-se como determinados projetos ou ferramentas estão funcionando e por quê. Verificam se há problemas, se modificações são necessárias e procuram explicar as relações de causa e efeito encontradas. Baseiam-se, fortemente, em observações de dados, entrevistas e material publicado. Necessitam, também, de mais de um site para análise comparativa, o que foi feito pelo estudo de dois treinamentos corporativos na web (Morra e Friedlander, 1999).

É importante notar que os estudos de casos múltiplos devem ser considerados como múltiplos experimentos, seguindo uma lógica de replicação, de modo que se resultados semelhantes são obtidos em todos os casos analisados, pode-se afirmar que os resultados foram replicados, ou que de fato ocorreu replicação (Yin, 1994:45).

Yin (1994:46) salienta que nos estudos de casos múltiplos cada caso deve ser cuidadosamente escolhido, de modo que produzam resultados similares ou contrastantes.

Seguindo essa recomendação, foi selecionada uma empresa (cuja identidade é omitida a pedido) e dois casos de treinamentos na web desenvolvidos por ela, com resultados finais contrastantes.

Um deles, denominado doravante de "programa A", foi considerado um sucesso pelo atingimento dos objetivos. O outro caso analisado envolve um treinamento desenvolvido pela mesma empresa, doravante denominado "programa B", o qual foi considerado um fracasso pela mesma, pelo não-atingimento dos objetivos.

De modo a atender às idéias de Yin (1994) necessárias à validação dos estudos de caso, considerou-se, cuidadosamente, os pontos relevantes para tal, quais sejam: validade da construção, validade interna, validade externa e confiabilidade.

\section{Validação da construção}

Para validação da construção, usou-se a tática de múltiplas fontes de dados e procurou-se estabelecer uma cadeia de evidências relativa às questões investigadas. Os próprios registros existentes relativos a esses projetos foram amplamente analisados e entrevistas com alguns dos envolvidos foram também realizadas. Assim, foram conduzidas entrevistas junto aos gestores de cada um dos programas de treinamento analisados. Essas entrevistas tiveram uma natureza "aberta", de modo a não apresentarem padrões previamente estabelecidos. Essa abordagem visou dar maior flexibilidade ao roteiro, permi- 
tindo, assim, a melhor exploração dos pontos analisados. Os gestores de cada programa foram identificados junto à empresa analisada, tendo sido apontado um único gestor para o primeiro caso ("programa A") e dois para o segundo caso ("programa B").

Além das entrevistas, foram aplicados questionários aos usuários dos programas de treinamento. Esses questionários visaram obter a sua percepção quanto às características de cada programa de treinamento, de acordo com as dimensões propostas pelo modelo de Reeves e Reeves (1997). Além disso, os usuários também forneceram sua percepção sobre o grau de atingimento dos objetivos propostos pelos programas de treinamento. Em ambos os casos foi usada escala de razão de 0 a 10.

No que tange às dimensões propostas por Reeves e Reeves (1997), o valor mínimo da escala (0) indica que a dimensão segue totalmente o paradigma behaviorista, enquanto o valor máximo da escala (10) indica uma dimensão totalmente associada ao paradigma construtivista. Já o valor máximo da escala (10) para atingimento de objetivo indica a percepção de sucesso absoluto do programa de treinamento, ao passo que o valor mínimo da mesma (0) indica uma total percepção de fracasso do programa de treinamento.

Foi determinado que para garantir a validade estatística das médias das avaliações dos programas de treinamento, cada uma das amostras deveria ter um mínimo de 30 casos, para que se pudesse aplicar o teorema do limite central da estatística (Sincich, 1995:513). Seguindo essa definição, os questionários foram respondidos por 32 usuários do primeiro programa analisado ("programa A") e 31 usuários do segundo ("programa B").

Após a análise inicial dos resultados, entrevistas não-estruturadas foram conduzidas com cinco diferentes usuários de cada programa. Essas entrevistas adicionais buscaram a compreensão e validação dos resultados quantitativos deste artigo, pela busca de dados qualitativos que os esclarecessem e corroborassem.

\section{Validação interna}

Segundo Yin (1994:35), a validade interna de um estudo de casos só deve ser uma preocupação do pesquisador quando o estudo tiver natureza causal ou explanatória, isto é, quando o investigador pretende determinar se o evento $x$ levou ao evento $y$.

Apesar da natureza preponderantemente exploratória deste artigo, a presença de elementos explanatórios torna necessária a descrição dos procedimentos que garantem a sua validade interna. 
Para determinar os fatores críticos de sucesso dos programa de treinamento analisados, foi realizada uma análise estatística dos dados obtidos nos questionários preenchidos pelos usuários. Os dados foram inicialmente tratados para eliminação de possíveis outliers, tendo sido usado, como ponto de corte, o valor correspondente a três desvios-padrão de afastamento em relação à média calculada (Kline, 1998:79). Não houve detecção de nenhum outlier no conjunto de dados de trabalho.

A primeira análise realizada visou a comparação da percepção dos usuários sobre o grau de atingimento dos objetivos propostos por cada um dos programas de treinamento, de modo a verificar se as respectivas médias das notas atribuídas pelos usuários poderiam ser consideradas estatisticamente diferentes. Para tanto, foi realizado um teste $t$ com nível de significância de 0,05 . Esse teste demonstrou que, segundo a percepção dos usuários, os resultados dos programas de treinamento foram efetivamente diferentes, e que o grau de atingimento dos objetivos do "programa A" foi maior que o grau de atingimento dos objetivos do "programa B", o que foi corroborado pela percepção de ambos os gestores dos programas.

Uma vez determinada a diferença entre o grau de atingimento dos objetivos de cada programa de treinamento, foi realizada uma comparação estatística das médias das percepções dos usuários para cada uma das dimensões do modelo de Reeves e Reeves (1997). Essa comparação também foi realizada utilizando teste $t$ com nível de significância de 0,05 , e teve como objetivo identificar as dimensões para as quais não houve diferença significativa de resultado entre os dois programas. Uma vez que já havia sido provado que os dois programas apresentaram diferença nos seus resultados, as dimensões que para ambos os programas não apresentaram diferenças estatisticamente significativas entre si foram descartadas como fatores críticos de sucesso.

Assim, da comparação anterior, foram descartadas duas dimensões do modelo de Reeves e Reeves (1997), restando oito dimensões a serem analisadas. Para isso, foi realizada uma regressão linear multivariada utilizando como variável dependente o grau de atingimento dos objetivos do programa de treinamento e como variáveis independentes as notas atribuídas pelos usuários a cada uma dessas dimensões. Foram analisados os níveis de significância de cada um dos coeficientes associados a cada uma das oito variáveis independentes (cada uma das oito dimensões analisadas), e foram descartados os coeficientes que não apresentavam evidência de correlação linear com a variável dependente (atingimento dos objetivos).

O resultado desse procedimento apontou as três dimensões que efetivamente podem ser consideradas fatores críticos de sucesso para os programas de treinamento analisados. 
Por fim, como validação quantitativa final, foi realizada uma regressão linear simples de cada uma das dimensões descartadas no atingimento dos objetivos. Essas regressões simples provaram que realmente não existia correlação linear forte entre essas dimensões e o atingimento dos objetivos.

A partir desses resultados, comparações qualitativas entre a percepção dos gestores e dos usuários foram analisadas, assim como foram realizadas entrevistas adicionais com cinco usuários de cada um dos programas de treinamento.

\section{Validação externa}

A validade externa está relacionada com a determinação de se os resultados das descobertas do trabalho podem ou não ser generalizados para outros casos similares não estudados (Yin, 1994:35). Neste artigo, estarão sendo estudados os mesmos fatores para dois casos distintos, numa mesma empresa, de modo a proporcionar validade externa à pesquisa, isto é, permitindo que os seus resultados possam ser aplicados a outros casos na mesma empresa.

\section{Confiabilidade}

Para garantir a confiabilidade deste estudo, foi utilizado um protocolo para a documentação dos procedimentos adotados. Além disso, um repositório de dados em formato digital foi criado para o armazenamento de todas as informações obtidas durante a coleta de dados.

Esse repositório contém a tabulação dos dados quantitativos obtidos durante a pesquisa de campo, além do resultado de todas as análises estatísticas efetuadas. Adicionalmente, armazenou-se uma cópia de todo material utilizado no referencial teórico para o qual foi possível obter-se uma versão digital.

\section{Limitações do método}

Yin (1994:9) aponta a falta de rigor da pesquisa, a tendenciosidade do pesquisador e a possível falta de base para generalização científica dos resultados e conclusões do estudo como os maiores alvos de crítica do método de estudo de casos. Este artigo, ao utilizar múltiplas fontes de dados, procura minimizar essas possíveis limitações do método. Além disso, o fato de se usar uma única empresa contribuiu consideravelmente para diminuir esses riscos. 
Mesmo considerando os procedimentos adotados para mitigar os possíveis problemas de pesquisa, é importante salientar que a extrapolação dos métodos aplicados e das conclusões propostas para outras empresas e programas de treinamento não traz garantias de obtenção dos mesmos tipos de resultados.

Adicionalmente, o uso de um marco teórico específico (Reeves e Reeves, 1997) pode ter trazido limitações ao método de pesquisa adotado. Isto é, o estudo não pode garantir que os fatores críticos de sucesso encontrados sejam os únicos existentes, mas sim aqueles deduzidos a partir do método e do referencial teórico aplicados.

\section{Descrição dos casos}

\section{A empresa}

A empresa analisada é uma multinacional de grande porte da área de tecnologia da informação, contando com mais de 300 mil funcionários, e escritórios em mais de 80 países de todos os continentes. A empresa atingiu em 2003 uma receita total de US\$ 85,9 bilhões e um lucro líquido (net income) de US\$ 7,6 bilhões.

Com uma presença geográfica tão abrangente, as restrições à implementação de programas de treinamento presenciais corporativos constituem um desafio permanente para a organização. Ao mesmo tempo, devido ao dinamismo dos mercados nos quais está inserida, existe uma grande necessidade de constante atualização tecnológica, de processos e legislação, entre outros temas igualmente relevantes para a execução dos negócios, demandando, assim, a implementação de programas de treinamento.

Nesse contexto foram idealizados e implementados os treinamentos corporativos a distância "programa A" e "programa B".

\section{O caso "programa A"}

Considerado um caso de sucesso pela empresa, o "programa A" é um programa de treinamento corporativo a distância, obrigatório a todos os seus gerentes, que são o público-alvo. Qualquer funcionário da empresa, em qualquer país do mundo, ao receber designação gerencial, é automaticamente cadastrado e tem que realizar esse curso no prazo máximo de um ano.

Segundo informações disponibilizadas na intranet da empresa, o "programa A" tem como objetivos ajudar os novos gerentes a construir conheci- 
mentos necessários para promover mudanças, alinhar comportamentos com as novas estratégias corporativas, reduzir a burocracia, enfatizar o foco nos clientes, e inspirar maior performance dos seus subordinados.

Esse programa de treinamento tem duração de nove meses e é composto por três fases distintas, que incluem treinamentos a distância e presenciais. $\mathrm{O}$ programa focaliza o desenvolvimento da liderança e é baseado no princípio de que o aprendizado é um processo contínuo ao longo da vida dos profissionais, e não um evento isolado. O "programa A" utiliza diversas tecnologias, entre as quais destaca-se a intranet para prover informações consideradas essenciais aos gerentes da empresa. O programa enfatiza as competências de liderança, estilos gerenciais, clima organizacional e coaching, entre outros aspectos ligados ao gerenciamento de pessoas. O programa inclui também a oportunidade para que o gerente obtenha um feedback de 360 graus em relação às suas habilidades de liderança.

A fase I (laboratório de pré-aprendizagem) é conduzida online, em um formato de treinamento a distância. Essa fase dura de cinco a seis meses, sendo uma atividade individual que requer de 48 a 56 horas do estudo.

A fase II (laboratório de aprendizagem) é uma experiência presencial de cinco dias. O comparecimento ao laboratório de aprendizagem da empresa é condicionado à conclusão bem-sucedida da fase I. Esse laboratório de aprendizagem é realizado no centro de aprendizagem mundial da empresa, nos EUA.

A fase III (laboratório de pós-aprendizagem), como a fase I, é realizada a distância. Essa fase é voltada para a aprendizagem colaborativa, utilizando a intranet da empresa, team room e ferramentas de instant messaging.

Durante todo o curso, um facilitador previamente designado está à disposição dos estudantes para solucionar qualquer dúvida, fornecer esclarecimentos ou ajudar a resolver problemas.

Os objetivos educacionais específicos definidos para o "programa A" são: desenvolver os melhores gerentes da empresa; prover informação e educação rapidamente para os gerentes da empresa; aperfeiçoar e fortalecer a liderança e o gerenciamento de pessoas por meio de e-learning, experiências de campo e coaching; e promover o aprendizado contínuo.

Segundo entrevista realizada junto ao gestor do "programa A", ele é considerado um sucesso, tendo atingido plenamente os seus objetivos.

Trinta e dois usuários do "programa A" responderam ao questionário e, em linhas gerais, avaliaram a experiência proporcionada pelo programa de treinamento como positiva (média de 8,50 com desvio-padrão de 1,32, em escala de razão de 0 a 10), considerando que seus objetivos foram atingidos. Todos os 32 usuários são gerentes da empresa no Brasil. 
Essa avaliação por parte dos usuários apresenta resultado compatível com a percepção do gestor, obtida com entrevista, corroborando, assim, o resultado positivo desse programa de treinamento.

\section{O caso "programa B"}

O "programa B" surgiu no início de 2000, inicialmente como um esforço para atender os requisitos de disponibilização de informações para os funcionários nos diferentes escritórios da empresa.

A definição e montagem deste programa foram realizadas pela área de TI (tecnologia da informação) da empresa, tendo como premissa básica a utilização da intranet para a disponibilização de conteúdo.

A primeira versão do programa foi composta pela consolidação das diferentes informações já publicadas na intranet, sob um "site único", com um índice único de pesquisa. Para tanto, foi montado um grupo de cinco funcionários provenientes de duas áreas de negócio distintas, para assessorar a área de TI na identificação e categorização das informações.

Uma vez identificadas e categorizadas as informações, a área de TI iniciou a implementação de ferramentas de avaliação e de "guias de uso", de modo a configurar diferentes cursos, categorizados por assunto, que poderiam ser acessados por qualquer usuário da empresa através da intranet. Para tanto, para cada curso a ser implementado, foi identificado um "gestor de programa", que passaria a ser responsável pela montagem das perguntas de avaliação (sempre do tipo múltipla escolha), e teria acesso aos seus resultados.

Não existia um critério preestabelecido para a identificação dos gestores, nem a definição de um perfil desejado para eles. Assim, os gestores foram selecionados, basicamente, de acordo com o conhecimento "técnico" do assunto específico de cada curso, e considerando-se sua disponibilidade para participar do processo. Os gestores selecionados não receberam treinamento específico relativo à montagem de programas de treinamento.

Além da ferramenta de avaliação, a área de TI disponibilizou um conjunto de profissionais especializados na criação de conteúdo multimídia para web (web designers), que poderia ser desenvolvido, por solicitação dos gestores, para enriquecimento do conteúdo de cada curso.

Após um período inicial de testes (com apenas um curso destinado a um grupo específico de usuários), foram disponibilizados três diferentes cursos, dos quais dois enfocavam a definição de processos específicos da empresa, e o terceiro enfocava conteúdo técnico.

Como pôde ser observado pelos pesquisadores durante entrevista realizada junto aos gestores desse programa de treinamento, não existiu uma ava- 
liação prévia de cada curso, dependendo da iniciativa de cada gestor a utilização ou não de teorias educacionais específicas. Aparentemente, acreditava-se que a simples publicação de conteúdo via intranet, possibilitando a consulta em qualquer horário por qualquer funcionário, seria suficiente para o sucesso do programa. Não existia, também, um professor ou facilitador que pudesse ser acessado pelos estudantes ao longo do curso. Eventualmente, os estudantes acessavam o gestor do curso que estavam fazendo, mas ele não desempenhava formalmente esse papel.

O objetivo primário do programa era diminuir os custos relacionados a treinamento, assim como diminuir o tempo de adaptação e capacitação de novos funcionários aos processos e padrões tecnológicos utilizados pela empresa. É importante salientar que, apesar desses objetivos genéricos, a definição clara dos objetivos específicos de cada curso dependia única e exclusivamente de seus gestores.

Depois de pouco menos de um ano de utilização, o programa, não tendo atingido seus objetivos, foi reestruturado.

Segundo entrevista realizada junto aos dois responsáveis pela implementação do programa, utilizando como referência formulário de pesquisa desenvolvido pelos autores, o programa de treinamento não atingiu seus objetivos inicialmente estabelecidos. Os dois gestores tiveram opiniões semelhantes quanto aos resultados obtidos, não deixando dúvidas sobre a avaliação negativa acerca do resultado do programa.

Trinta e um usuários do "programa B" responderam ao questionário e, em linhas gerais, avaliaram a experiência proporcionada pelo programa de treinamento como negativa, considerando que os objetivos não foram atingidos (média de 4,52 com desvio-padrão de 1,15, em escala de razão de 0 a 10). Todos os usuários que responderam ao questionário são funcionários da empresa no Brasil.

Essa avaliação por parte dos usuários apresenta resultado compatível com a avaliação dos seus gestores, corroborando, assim, o não-atingimento dos objetivos por esse programa de treinamento. É interessante ressaltar que, em entrevistas informais conduzidas com cinco usuários após a avaliação inicial dos resultados, foi explicitamente comentada a falta de interesse e motivação, não só para participar do treinamento, mas, também, para propor ou recomendar qualquer mudança que viesse a melhorar o programa.

\section{Comparação de resultados}

A comparação dos casos apresentados neste artigo identifica as efetivas causas para o atingimento ou não dos objetivos dos programas, de modo a permitir a 
inferência dos fatores-chave de sucesso para a implementação de programas de treinamento corporativos a distância baseados na web.

Primeiramente, é fundamental salientar uma diferença básica identificada na montagem de cada um dos programas de treinamento. O "programa A" foi precedido de uma fase inicial de planejamento que procurou identificar as melhores práticas, metodologias e referencial teórico para a sua implementação, considerando, assim, suas características específicas. Conforme apresentado no referencial teórico deste artigo, o planejamento prévio, em especial a determinação dos objetivos, pode ser identificado como um dos fatores-chave de sucesso para os programas de treinamento (Dick e Carey, 1996; Kay, Dodd e Sime, 1970; Mager, 1972; Sancho, 1998). Mais especificamente, para os programas de treinamento a distância, é necessário avaliar os objetivos do programa, as teorias de aprendizado que podem ser utilizadas e, por fim, as tecnologias que podem ser aplicadas na sua construção (Wilhelmsen, Åsmul e Meistad, 1998).

Além desses fatores, a análise das características dos programas de treinamento segundo o modelo de Reeves e Reeves (1997) ajuda a identificar outros fatores-chave de sucesso desse tipo de empreendimento. Apesar de o modelo teórico não poder ser utilizado para avaliar se um determinado conjunto de características é melhor do que outro para um determinado programa de treinamento, a análise de algumas das diferenças encontradas na comparação dos programas aqui analisados contribui para a identificação desses fatores.

Primeiramente, é necessário analisar as diferenças apontadas pelos gestores e usuários quanto ao atingimento dos objetivos do programa de treinamento. Na avaliação dos gestores do "programa A", os objetivos foram plenamente atingidos, e a avaliação geral do programa foi classificada como "muito boa". Já na avaliação dos gestores do "programa B", os resultados não foram atingidos, e o programa acabou por ser reestruturado. Na comparação da avaliação dos gestores, portanto, fica clara a diferença no grau de atingimento dos objetivos entre os dois programas.

Para analisar a diferença de percepção dos usuários, é necessário avaliar a diferença das médias das notas atribuídas por eles a cada um dos programas. A média da avaliação (de 0 a 10) quanto ao atingimento dos objetivos dos usuários do "programa A" foi $8,50(s=1,32 ; n=32)$, enquanto a dos usuários do "programa B" foi 4,52 ( $s=1,15 ; n=31)$. Essa diferença nas médias das avaliações parece corroborar a opinião dos gestores dos programas. É necessário, no entanto, realizar um teste estatístico de comparação das médias das amostras correspondentes a cada programa (teste $t$ ) para verificar se elas podem, efetivamente, ser consideradas diferentes a determinado nível de significância.

A tabela 1 apresenta o resultado do teste de comparação das médias das avaliações dos usuários de cada um dos programas analisados quanto ao atingimento dos objetivos. 
Tabela 1

Comparação das médias de atingimento dos objetivos, segundo os usuários dos programas de treinamento

\begin{tabular}{|c|c|c|c|c|c|c|c|c|c|}
\hline & \multicolumn{2}{|c|}{$\begin{array}{l}\text { Teste de Levene } \\
\text { de igualdade de } \\
\text { variâncias }\end{array}$} & \multicolumn{7}{|c|}{ Teste t para igualdade de médias } \\
\hline & \multirow[b]{2}{*}{$\mathrm{F}$} & \multirow[b]{2}{*}{ Sig. } & \multirow[b]{2}{*}{$\mathrm{t}$} & \multirow{2}{*}{ df } & \multirow{2}{*}{$\begin{array}{c}\text { Sig. } \\
\text { (2 caudas) }\end{array}$} & \multirow{2}{*}{$\begin{array}{l}\text { Diferença } \\
\text { média }\end{array}$} & \multirow{2}{*}{$\begin{array}{c}\text { Diferença } \\
\text { de desvio- } \\
\text { padrão }\end{array}$} & \multicolumn{2}{|c|}{$\begin{array}{l}\text { Intervalo da diferença } \\
\text { com } 95 \% \text { de confiança }\end{array}$} \\
\hline & & & & & & & & Inferior & Superior \\
\hline Atingimento & & & & & & & & & \\
\hline dos objetivos & 0,202 & 0,655 & 12,752 & 61 & 0,000 & 3,98 & 0,31 & 3,36 & 4,61 \\
\hline
\end{tabular}

A partir dos resultados apresentados na tabela 1 constata-se que, com 95\% de confiança, existe uma diferença significativa entre as médias da avaliação dos usuários quanto ao atingimento dos objetivos dos programas de treinamento. Além disso, observando que o intervalo de confiança é positivo, é possível determinar que a média do "programa A" é maior que a média do "programa B" (Sincich, 1995:532).

A partir da análise comparativa das médias das avaliações dos usuários de cada programa e da constatação da similaridade dessa avaliação com a opinião dos seus respectivos gestores, pode-se efetivamente afirmar que, em termos de objetivos atingidos, o "programa A" teve resultados melhores que o "programa B".

A partir da determinação desse fato, pode-se buscar a identificação dos fatores que influenciaram esses resultados, segundo o modelo proposto neste artigo. Para isso, foram analisadas as médias das avaliações de cada uma das dimensões do modelo de Reeves e Reeves (1997), procurando identificar quais delas efetivamente influenciaram os diferentes resultados obtidos.

Analogamente, nas dimensões onde existe uma diferença significativa entre a média da amostra de cada programa de treinamento, pode-se considerar a dimensão como tendo papel influente no resultado do atingimento dos objetivos. A tabela 2, a seguir, apresenta a comparação das médias para cada uma das dimensões do modelo de Reeves e Reeves (1997). 


\begin{tabular}{|c|c|c|c|c|c|c|c|c|c|}
\hline \multicolumn{10}{|c|}{$\begin{array}{c}\text { Tabela } 2 \\
\text { Comparação das médias das dimensões do modelo das amostras }\end{array}$} \\
\hline \multirow{3}{*}{ Dimensão } & \multicolumn{3}{|c|}{$\begin{array}{l}\text { Teste de Levene } \\
\text { de igualdade de } \\
\text { variâncias }\end{array}$} & \multicolumn{6}{|c|}{ Teste t para igualdade de médias } \\
\hline & \multirow[b]{2}{*}{$\mathrm{F}$} & \multirow[b]{2}{*}{ Sig. } & \multirow[b]{2}{*}{$\mathrm{t}$} & \multirow{2}{*}{ df } & \multirow{2}{*}{$\begin{array}{c}\text { Sig. } \\
\text { (2 caudas) }\end{array}$} & \multirow{2}{*}{$\begin{array}{l}\text { Diferença } \\
\text { média }\end{array}$} & \multirow{2}{*}{$\begin{array}{l}\text { Diferença } \\
\text { de desvio- } \\
\text { padrão }\end{array}$} & \multicolumn{2}{|c|}{$\begin{array}{l}\text { Intervalo da diferença } \\
\text { com } 95 \% \text { de confiança }\end{array}$} \\
\hline & & & & & & & & Inferior & Superior \\
\hline $\begin{array}{l}\text { Filosofia } \\
\text { pedagógica }\end{array}$ & 0,010 & 0,919 & 0,511 & 61 & 0,611 & 0,11 & 0,23 & $-0,34$ & 0,56 \\
\hline $\begin{array}{l}\text { Teoria de } \\
\text { aprendizado }\end{array}$ & 55,065 & 0,000 & 2,470 & 61 & 0,016 & 0,52 & 0,21 & 0,09 & 0,94 \\
\hline $\begin{array}{l}\text { Orientação dos } \\
\text { objetivos }\end{array}$ & 4,285 & 0,043 & 6,239 & 61 & 0,000 & 1,36 & 0,22 & 0,92 & 1,79 \\
\hline $\begin{array}{l}\text { Orientação } \\
\text { das tarefas }\end{array}$ & 16,813 & 0,000 & 4,963 & 61 & 0,000 & 1,03 & 0,21 & 0,61 & 1,44 \\
\hline $\begin{array}{l}\text { Fonte de } \\
\text { motivação }\end{array}$ & 8,686 & 0,005 & 4,951 & 61 & 0,000 & 1,15 & 0,23 & 0,68 & 1,61 \\
\hline $\begin{array}{l}\text { Papel do } \\
\text { professor }\end{array}$ & 28,837 & 0,000 & 6,790 & 61 & 0,000 & 2,56 & 0,38 & 1,81 & 3,31 \\
\hline $\begin{array}{l}\text { Suporte } \\
\text { metacognitivo }\end{array}$ & 68,946 & 0,000 & 9,747 & 61 & 0,000 & 1,94 & 0,20 & 1,54 & 2,33 \\
\hline $\begin{array}{l}\text { Aprendizado } \\
\text { colaborativo }\end{array}$ & 129,092 & 0,000 & 3,760 & 61 & 0,000 & 0,78 & 0,21 & 0,37 & 1,20 \\
\hline $\begin{array}{l}\text { Sensibilidade } \\
\text { cultural }\end{array}$ & 20,583 & 0,000 & 7,756 & 61 & 0,000 & 1,00 & 0,13 & 0,74 & 1,26 \\
\hline $\begin{array}{l}\text { Flexibilidade } \\
\text { estrutural }\end{array}$ & 0,943 & 0,335 & $-0,751$ & 61 & 0,455 & $-0,19$ & 0,26 & $-0,71$ & 0,32 \\
\hline
\end{tabular}

Como pode ser observado a seguir, com 95\% de confiança, as dimensões filosofia pedagógica e flexibilidade estrutural não são estatisticamente diferentes entre os casos analisados, podendo, dessa forma, ser descartadas da análise dos fatores críticos de sucesso. A partir desse resultado, foi realizada uma regressão linear múltipla do atingimento dos objetivos (variável dependente) em relação às oito dimensões que apresentaram médias significativamente diferentes (variáveis independentes), para se verificar quais variáveis poderiam ser consideradas como efetivamente influentes nos resultados obtidos. É importante salientar que essa regressão linear não objetiva a previsão do resultado de outros programas a partir das dimensões do modelo de Reeves 
e Reeves (1997), mas sim verificar a influência das variáveis nos resultados dos programas analisados.

A tabela 3 apresenta o sumário do modelo estatístico obtido para essa regressão.

\section{Tabela 3}

Resumo da regressão linear das dimensões do modelo

\begin{tabular}{ccccc}
\hline Modelo & $\mathrm{R}$ & $\mathrm{R}^{2}$ & $\mathrm{R}^{2}$ ajustado & Desvio-padrão da estimativa \\
\hline 1 & $0,847^{*}$ & 0,717 & 0,675 & 1,34
\end{tabular}

* Previsores (constantes): sensibilidade cultural, teoria de aprendizado, fonte de motivação, orientação dos objetivos, papel do professor, orientação das tarefas, aprendizado colaborativo, suporte metacognitivo.

Este resumo comprova a validade da utilização das oito dimensões indicadas (predictors) para a previsão do atingimento dos objetivos para os casos estudados (no resumo, a coluna $\mathrm{R}$ representa o coeficiente de correlação e a coluna $\mathrm{R}^{2}$ o coeficiente de determinação). Pelos dados apresentados, podese afirmar que aproximadamente $70 \%$ do resultado da variável atingimento dos objetivos são explicados pelas dimensões incluídas na regressão.

A partir da verificação da validade desse modelo, deve-se verificar cada um dos seus coeficientes (correspondentes às dimensões do modelo incluídas), de modo a determinar quais são efetivamente influentes na determinação do atingimento dos objetivos. A tabela 4 apresenta o resumo das estatísticas relacionadas aos coeficientes nesse modelo de regressão.

Observando os resultados apresentados na tabela 4, nota-se que com 95\% de confiança, as dimensões teoria de aprendizado, orientação das tarefas, papel do professor, aprendizado colaborativo e sensibilidade cultural não apresentam evidências de relação linear significativa com o atingimento dos objetivos.

\begin{tabular}{|c|c|c|c|c|c|c|c|c|c|}
\hline \multicolumn{10}{|c|}{$\begin{array}{c}\text { Tabela } 4 \\
\text { Análise de significância estatística dos coeficientes da regressão } \\
\text { linear das dimensões do modelo* }\end{array}$} \\
\hline \multirow{2}{*}{ Modelo 1} & \multicolumn{2}{|c|}{$\begin{array}{l}\text { Coeficientes não- } \\
\text { padronizados }\end{array}$} & \multirow{2}{*}{$\begin{array}{c}\text { Coeficientes } \\
\text { padronizados } \\
\text { Beta }\end{array}$} & \multirow[b]{2}{*}{$t$} & \multirow[b]{2}{*}{ Sig. } & \multicolumn{2}{|c|}{$\begin{array}{l}\text { Intervalo com 95\% } \\
\text { de confiança para B }\end{array}$} & \multicolumn{2}{|c|}{$\begin{array}{l}\text { Estatística de } \\
\text { colinearidade }\end{array}$} \\
\hline & B & $\begin{array}{l}\text { Erro- } \\
\text { padrão }\end{array}$ & & & & $\begin{array}{l}\text { Limite } \\
\text { inferior }\end{array}$ & $\begin{array}{l}\text { Limite } \\
\text { superior }\end{array}$ & Tolerância & FIV \\
\hline (Constante) & 2,160 & 0,547 & & 3,950 & 0,000 & 1,063 & 3,256 & & \\
\hline Teoria de aprendizado & $-4,589 E-021$ & 0,230 & $-0,017$ & $-0,200$ & 0,843 & $-0,507$ & 0,415 & 0,727 & 1,376 \\
\hline Orientação dos objetivos & 0,486 & 0,211 & 0,226 & 2,299 & 0,025 & 0,062 & 0,910 & 0,541 & 1,849 \\
\hline Orientação das tarefas & $-0,215$ & 0,256 & $-0,088$ & $-0,839$ & 0,405 & $-0,729$ & 0,299 & 0,475 & 2,105 \\
\hline
\end{tabular}




\begin{tabular}{|c|c|c|c|c|c|c|c|c|c|}
\hline \multirow{2}{*}{ Modelo 1} & \multicolumn{2}{|c|}{$\begin{array}{l}\text { Coeficientes não- } \\
\text { padronizados }\end{array}$} & \multirow{2}{*}{$\begin{array}{c}\begin{array}{c}\text { Coeficientes } \\
\text { padronizados }\end{array} \\
\text { Beta }\end{array}$} & \multirow[b]{2}{*}{$t$} & \multirow[b]{2}{*}{ Sig. } & \multicolumn{2}{|c|}{$\begin{array}{l}\text { Intervalo com 95\% } \\
\text { de confiança para B }\end{array}$} & \multicolumn{2}{|c|}{$\begin{array}{l}\text { Estatística de } \\
\text { colinearidade }\end{array}$} \\
\hline & B & $\begin{array}{l}\text { Erro- } \\
\text { padrão }\end{array}$ & & & & $\begin{array}{l}\text { Limite } \\
\text { inferior }\end{array}$ & $\begin{array}{l}\text { Limite } \\
\text { superior }\end{array}$ & Tolerância & FIV \\
\hline Fonte de motivação & 0,845 & 0,209 & 0,388 & 4,046 & 0,000 & 0,426 & 1,263 & 0,571 & 1,753 \\
\hline Papel do professor & 0,100 & 0,124 & 0,084 & 0,805 & 0,424 & $-0,149$ & 0,349 & 0,486 & 2,058 \\
\hline Suporte metacognitivo & 0,645 & 0,228 & 0,342 & 2,833 & 0,006 & 0,189 & 1,101 & 0,359 & 2,785 \\
\hline Aprendizado colaborativo & 0,108 & 0,271 & 0,042 & 0,399 & 0,691 & $-0,436$ & 0,652 & 0,478 & 2,090 \\
\hline Sensibilidade cultural & 0,288 & 0,384 & 0,087 & 0,750 & 0,457 & $-0,481$ & 1,057 & 0,387 & 2,587 \\
\hline
\end{tabular}

Um dado importante, também apresentado na tabela 4, é a verificação da eventual multicolinearidade das variáveis deste modelo de regressão. A coluna FIV (fator de inflação da variância) indica que o modelo não apresenta multicolinearidade, já que os valores para cada uma das variáveis são menores que 10 (Hair Jr. et al., 1998:193).

Para corroborar os resultados desta regressão múltipla quanto à falta de evidências da relação linear das dimensões teoria de aprendizado, orientação das tarefas, papel do professor, aprendizado colaborativo e sensibilidade cultural, foi realizada uma regressão linear simples de cada uma dessas variáveis com o atingimento dos objetivos. A tabela 5 apresenta o resumo dos modelos das quatro regressões simples.

\begin{tabular}{|c|c|c|c|c|}
\hline \multicolumn{5}{|c|}{$\begin{array}{c}\text { Tabela } 5 \\
\text { Sumário dos modelos de regressão linear simples } \\
\text { das variáveis descartadas na regressão múltipla }\end{array}$} \\
\hline Modelo & $\mathrm{R}$ & $\mathrm{R}^{2}$ & $\mathrm{R}^{2}$ ajustado & $\begin{array}{l}\text { Erro-padrão } \\
\text { da estimativa }\end{array}$ \\
\hline $1^{\mathrm{a}}$ & $0,291^{a}$ & 0,085 & 0,070 & 2,27 \\
\hline $2^{b}$ & $0,494^{a}$ & 0,244 & 0,232 & 2,06 \\
\hline $3^{c}$ & $0,524^{a}$ & 0,275 & 0,263 & 2,02 \\
\hline $4^{\mathrm{d}}$ & $0,462^{\mathrm{a}}$ & 0,213 & 0,200 & 2,11 \\
\hline $5^{e}$ & $0,514^{a}$ & 0,265 & 0,253 & 2,04 \\
\hline \multicolumn{5}{|c|}{$\begin{array}{l}\text { a Previsores (constante): teoria de aprendizado. } \\
\text { o Previsores (constante): aprendizado colaborativo. } \\
\text { ' Previsores (constante): orientação das tarefas. } \\
\text { d Previsores (constante): papel do professor. } \\
\text { e Previsores (constante): sensibilidade cultural. }\end{array}$} \\
\hline
\end{tabular}


Como pode ser observado pela análise do coeficiente de correlação (coluna $\mathrm{R}$ ) e do coeficiente de determinação (coluna $\mathrm{R}^{2}$ ) dos modelos de regressão simples, essas variáveis efetivamente não apresentam uma forte relação com a variável atingimento dos objetivos $\left(\mathrm{R}^{2}\right.$ menor que 0,3$)$.

Por fim, uma última análise estatística deve ser realizada. Analisando o resultado da regressão linear múltipla das três variáveis selecionadas como fatores influentes no atingimento dos objetivos dos programas de treinamento (orientação dos objetivos, fonte de motivação e suporte metacognitivo), observa-se que este último modelo é similar ao modelo de regressão múltipla anterior, que considerava oito variáveis. A tabela 6 apresenta o resumo desse modelo.

\begin{tabular}{|lcccc|}
\hline \multicolumn{4}{|c|}{ Tabela 6} \\
Sumário da regressão linear das dimensões suporte \\
metacognitivo, fonte de motivação e orientação dos objetivos \\
\hline Modelo & $\mathrm{R}$ & $\mathrm{R}^{2}$ & $\mathrm{R}^{2}$ ajustado & $\begin{array}{r}\text { Erro-padrão } \\
\text { da estimativa }\end{array}$ \\
\hline 1 & $0,838^{*}$ & 0,703 & 0,688 & 1,32 \\
\hline * Previsores (constantes): suporte metacognitivo, fonte de motivação, orientação dos objetivos.
\end{tabular}

\section{Considerações finais}

A partir dos testes estatísticos de comparação de médias e da verificação de relação linear das dimensões do modelo de Reeves no atingimento dos objetivos, pode-se chegar à conclusão que as dimensões especialmente influentes e que caracterizaram mais fortemente o resultado dos programas de treinamento analisados são a orientação dos objetivos, a fonte de motivação e o suporte metacognitivo.

As baixas médias observadas para a dimensão orientação dos objetivos (2,94 para o "programa A" e 1,58 para o "programa B") indicam que os objetivos dos dois programas eram mais específicos do que genéricos.

Pela falta de uma definição formal acerca dos objetivos do "programa B", foi constatado nas entrevistas com cinco usuários do mesmo, que eles percebiam objetivos diferentes entre si, o que parece ter contribuído para o nãoatingimento dos objetivos do programa. Por outro lado, no caso do "programa A", os objetivos estavam claramente definidos para os usuários, de modo que houve congruência de percepção pelos usuários. 
Com média de 1,06, o "programa B" praticamente não implementa suporte metacognitivo, enquanto o "programa A", com média 3,00, apresenta um certo nível de implementação. Novamente recorrendo a dados coletados em entrevistas informais, usuários do "programa B" afirmaram que não existia uma ferramenta para o acompanhamento, pelo estudante, de seu progresso ao longo dos diferentes cursos oferecidos.

Ainda sobre o suporte metacognitivo, a própria descrição dos gestores das facilidades oferecidas aos usuários pelo "programa B" (que se resumiam ao acesso via intranet e a questionários de múltipla escolha) demonstra e atesta a falta de meios para que os usuários desse programa pudessem avaliar de forma contínua suas estratégias de aprendizado.

O "programa A", por outro lado, efetivamente oferecia algumas oportunidades para que os usuários realizassem esse tipo de avaliação. A ferramenta sobre a qual foi construído o programa de treinamento a distância permitia que os usuários acompanhassem os resultados obtidos em cada etapa do programa, o percentual já utilizado do tempo total estimado para completar o curso, e o tempo total estimado para a conclusão de cada etapa. Além disso, o "programa A" permitia que os próprios usuários marcassem os conteúdos recomendados como já utilizados, ajudando-os a manter o controle sobre o processo de aprendizado.

Por fim, a avaliação dos usuários do "programa B", com relação à dimensão fonte de motivação, apresentou média de 1,26 , indicando que a motivação dos estudantes era eminentemente extrínseca. Ainda que os resultados do "programa A" também apontem para esse extremo do continuum (média 2,41 ) fica claro que, nesse último caso, existe ao menos alguma motivação intrínseca referente ao programa de treinamento em si. Também é fundamental recordar que existe uma motivação prévia de seus usuários, uma vez que estes haviam sido recentemente promovidos a um cargo gerencial. Dessa forma, pode-se considerar que os usuários do "programa A" estavam motivados, se não a partir do curso em si, ao menos com a empresa e com suas carreiras, informação confirmada pela entrevista realizada junto a cinco dos usuários desse programa de treinamento.

Os usuários do "programa B", por outro lado, pareciam não possuir qualquer motivação para participar do programa de treinamento, a não ser a sua natureza compulsória. Essa falta de motivação também foi confirmada nas entrevistas realizadas com os cinco usuários do programa, que atestaram, por exemplo, que "só participavam do curso porque eram obrigados a fazê-lo".

Assim, da análise do referencial teórico e da comparação dos dois casos estudados, podem ser considerados fatores-chave de sucesso para a implementação de programas de treinamento corporativos a distância via web: 
จ a definição clara do conteúdo, público-alvo e objetivos do programa de treinamento;

$\checkmark$ a motivação dos usuários;

v a implementação no ambiente web de um nível adequado de suporte metacognitivo.

É interessante observar que os resultados corroboraram as idéias de Ertmer e Newby (1993) de que a seleção de uma abordagem de aprendizado específica não representa por si só um fator crítico de sucesso para o treinamento. Além disso, a verificação de que essa dimensão não influenciou diretamente o resultado obtido por cada um dos programas analisados (uma vez que os dois programas possuíam características behavioristas), é coerente com a proposição do modelo de Reeves e Reeves (1997), no sentido de que ele não pretende defender que programas mais behavioristas ou mais construtivistas sejam, necessariamente, melhores do que outros.

Também é relevante salientar que a comprovação da importância da orientação dos objetivos apontada na análise comparativa dos casos é coerente com o apresentado no referencial teórico deste artigo, que aponta a importância da definição clara dos objetivos para os programas de treinamento (Kay, Dodd e Sime, 1970; Mager, 1972; Dick e Carey, 1996; Sancho, 1998).

Por outro lado, a possibilidade de recapitulação das estratégias dos estudantes em qualquer ponto do processo de resolução de problemas é fundamental para que o suporte metacognitivo esteja presente em programas de treinamento baseados na web, como realmente atestam Campbell e outros (2000).

Adicionalmente, comprovou-se que a motivação é um fator primário em qualquer teoria ou modelo de aprendizado, com o qual também corroboram Carroll (1968) e Amabile (1993).

É importante salientar que não existe um modelo ou uma fórmula única para garantir o sucesso dos programas de treinamento corporativos a distância. Cada empresa e cada programa de treinamento é um problema único, com características específicas que devem ser observadas, para que seja possível adotar as estratégias corretas e atingir os objetivos desejados. No caso do "programa A", por exemplo, foi considerado imprescindível pelos idealizadores do programa a realização de uma classe presencial logo após a fase de treinamento a distância, como forma de consolidar os conceitos apresentados ao longo da primeira fase do curso.

Dessa forma, este artigo atingiu seu objetivo que foi identificar os critérios de sucesso na construção de programas de treinamento corporativos a distância via web, a partir dos dois estudos de casos analisados. A figura que 
ilustra o artigo demonstra, de forma resumida, a inter-relação e a influência dos fatores críticos de sucesso para a elaboração de programas de treinamento a distância via web, segundo os resultados obtidos nessa pesquisa.

Como sugere a figura, os três fatores de sucesso derivados das inferências das avaliações baseadas no modelo de Reeves e Reeves (1997) fornecem a base de sustentação para a seleção da teoria de aprendizado que será utilizada, para a determinação das estratégias de design instrucional e para a seleção de tecnologias para sua implementação. Esses fatores, por sua vez, são a base para o programa de treinamento em si. É também importante notar que o modelo todo possui retroalimentação, ou seja, os fatores interagem entre si ao longo do ciclo de vida do programa de treinamento.

\section{Fatores críticos de sucesso para os programas de treinamento a distância via web}

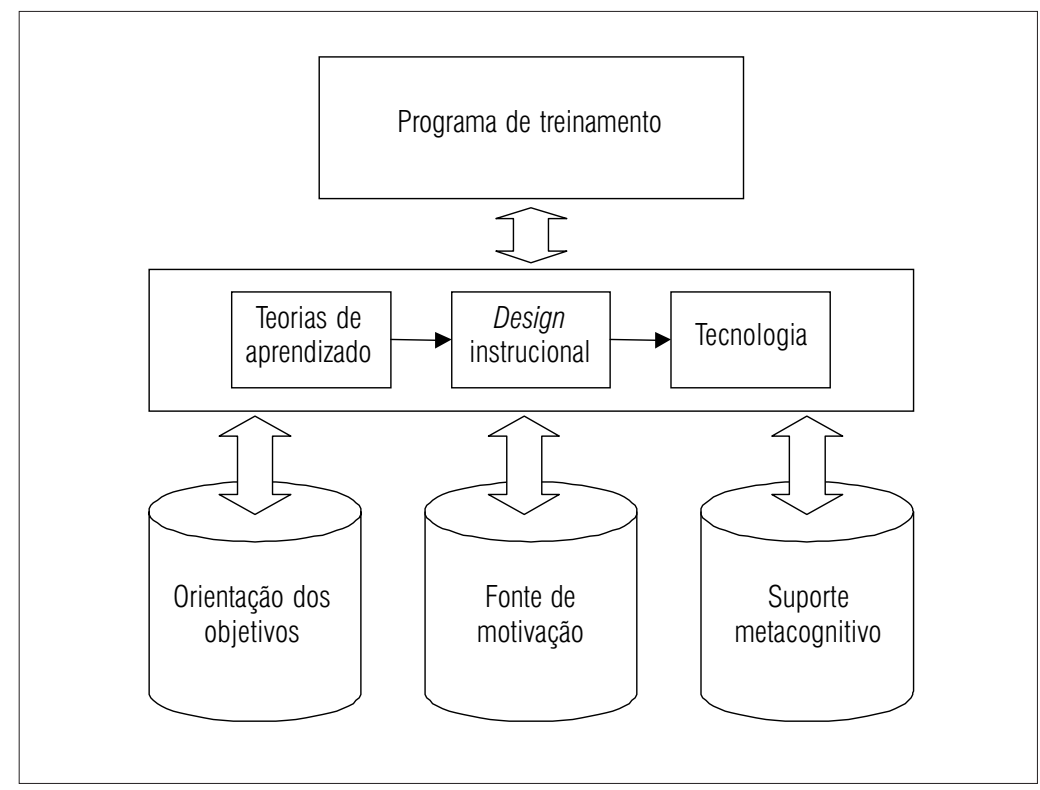

Este artigo não pretendeu ser um tratado definitivo sobre o tema em questão. O assunto merece, certamente, mais estudos. Pesquisas envolvendo um número maior de empresas e focando em cada um dos aspectos específicos do processo de criação de programas de treinamento a distância via web poderiam, certamente, revelar outros fatores importantes e contribuir para que os 
programas de treinamento a distância via web das grandes empresas possam ser aprimorados e seus benefícios efetivamente medidos.

Além disso, permanece em aberto a trilha de pesquisa que visa avaliar o return on training (ROT) de treinamentos corporativos a distância na web, que, se medido, permitiria às empresas compreender a real eficácia e impacto de programas dessa natureza na sua performance corporativa.

\section{Referências bibliográficas}

ABUHEJLEH, A. New ways of thinking about multimedia and online teaching in higher education. 2002. University of Wisconsin. Disponível em: <www.cs.uni.edu/ fienup/ mics_2002/proceedings/papers/ABUHEJLE.pdf>. Acesso em: jul. 2005.

AMABILE, T. Motivating creativity in organizations. California Management Review, v. 40, n. 1, p. 39-58, Fall 1993.

BATES, A. W. Technology, open learning and distance education. London and New York: Routledge, 1995.

CAMPBELL, D. M. et al. Portfolio and performance assessment in teacher education. New York: Allyn \& Bacon, 2000.

CAREY, T. et al. Effectiveness of learning technologies: the costs and effectiveness of technology-based approaches to teaching and learning. Ontario: University of Guelph, 1998.

CARROLL, J. B. On learning from being told. Educational Psychologist, n. 5, p. 4-10, 1968.

CLARK, R. E. Reconsidering research on learning from media. Review of Educational Research, v. 53, n. 4. p. 445-459, 1983.

COLE, P. Constructivism revisited: a search for common ground. Educational Technology, v. 32, n. 2, p. 27-34, 1992.

DICK, W.; CAREY, L. The systematic design of instruction. New York: HarperCollins Publisher, 1996.

DUFFY, T.; JONASSEN, D. H. Constructivism and the technology of instruction: a conversation. Hillsdale, NJ: Lawrence Erlbaum, 1992.

EISENHARDT, K. M. Building theories from case study research. Academy of Management Review, v. 14, n. 4, p. 532-550, 1989. 
ERTMER, P. A.; NEWBY, T. J. Behaviorism, cognitivism, constructivism: comparing critical features from an instructional design perspective. 1993. Disponível em: <http:// vcs.ccc.cccd.edu/crs/special/ertnew1.htm>. Acesso em: set. 2005.

HAIR JR., J. F. et al. Multivariate data analysis. 5. ed. Upper Saddle River, NJ: Prentice Hall, 1998.

JOIA, L. A. Evaluation of hybrid socio-construtivist model for teacher training. Journal of Technology and Teacher Education, v. 9, n. 4, p. 519-549, 2001.

KAY, H.; DODD, B.; SIME, M. Iniciação à instrução programada e às máquinas de ensinar. São Paulo: Ibrasa, 1970.

KLINE, R. B. Principles and practice of structural equation modeling. New York: The Guilford Press, 1998.

MAGER, R. F. Objetivos para o ensino efetivo. Rio de Janeiro: Senai, 1972.

MARTIN, K. WBI or not WBI? Issues of Teaching and Learning, v. 4, n. 7, Aug. 1998. Disponível em: <www.catl.uwa.edu.au/NEWSLETTER/issue0798/dimensions.html>. Acesso em: set. 2005.

MERRIAM, S. B. An update on adult learning theory. San Francisco: Jossey-Bass, 1993.

MORRA, L.; FRIEDLANDER, A. C. Case study evaluations. Operations Evaluation Department, Working Paper Series n. 2, World Bank, May 1999.

PAPERT, S. The children's machine: rethinking school in the age of the computer. New York: Basic Books, 1993.

PENUEL, B.; ROSCHELLE, J. Designing learning: cognitive science principles for the innovative organization. Menlo Park, CA: Center for Technology in Learning. SRI International, 1999.

PIAGET, J. The origins of intelligence in children. New York: International Universities Press, Inc., 1952.

POWELL, G. C. Are you ready for WBT? 2000. Wayne State University. Disponível em: <http://it.coe.uga.edu/itforum/paper39/paper39.html>. Acesso em: set. 2005.

REEVES, T. C. Evaluating what really matters in computer-based education. 1996. Georgia University. Disponível em: <www.educationau.edu.au/archives/cp/reeves.htm>. Acesso em: set. 2005.

. A model of the effective dimensions of interactive learning on the world wide web. 1997. Georgia Unversity. Disponível em: <http://it.coe.uga.edu/ treeves/ WebPaper.pdf>. Acesso em: set. 2005. 
. A model to guide the integration of the WWW as a cognitive tool in the K-12 education. 1999. Georgia University. Disponível em: <http://it.coe.uga.edu/ treeves/ AERA99Web.pdf>. Acesso em: dez. 2005.

; REEVES, M. Effective dimensions of interactive learning on the world wide web. In: KHAN, B. (Ed.). Web-based instruction. Englewood Cliffs, NJ: Educational Technology Publications, 1997.

RIEBER, L. P. Computer-based microworlds: a bridge between constructivism and direct instruction. Educational Technology Research and Development, v. 40, n. 1, p. 93-106, 1992.

ROSEMBERG, M. J. E-learning - strategies for delivering knowledge in the Digital Age. New York: McGraw-Hill, 2001.

SANCHO, J. M. Para uma tecnologia educacional. Porto Alegre: ArtMed, 1998.

SKINNER, B. F. Technology of teaching. New York: Meredith Publishing, 1968.

SINCICH, T. Business statistics by example. New Jersey: Prentice Hall, 1995.

UNIVERSITY OF DAYTON. Beginning instructional design. 2003. Williams e-Learning Lab. Disponível em: <http://academic.udayton.edu/elearning/onlineTraining/ InstructionalDesign/>. Acesso em: fev. 2005.

WILHELMSEN, S.; ÅSMUL, S. I.; MEISTAD, Ø. Psychological theories; a brief survey of the changing views of learning. 1998. Disponível em: <www.uib.no/People/sinia/CSCL/ web_struktur-4.htm>. Acesso em: fev. 2005.

YIN, R. K. Case study research: design and methods. 2. ed. California: Sage Publications, 1994. 